\title{
Peripheral Nervous System Involvement in Diabetes and Role of Rehabilitation
}

\section{William Jens and Aiesha Ahmed*}

Department of Neurology, Penn State University, 30 Hope Drive, EC 037 Hershey, PA 17033, USA

\begin{abstract}
Diabetes is well known to affect peripheral nervous system. We have summarized the multiple different presentations of peripheral nervous system involvement in this disease along with the medical and rehabilitation management. The clinical presentation depends on the localization of the damage which can involve nerve roots, plexus, focal nerves, diffuse involvement of multiple nerves and autonomic system. The process can be acute or insidious based on the pathology which can be variable.
\end{abstract}

Keywords: Numbness; Neuropathy; Hyperglycemia

\section{Introduction}

Diabetes neuropathy $(\mathrm{DN})$ is a growing concern with increased morbidity and mortality. The swings in blood sugar and high blood sugar damage both the vasculature and the nerves contributing to the disease and depending on the sites of injuries, can manifest with four different presentations, peripheral, focal, proximal and autonomic. Initial evaluation can consist of thorough history and physical exam with laboratory testing, electromyography, biopsy and imaging if indicated. Treatment is focused on strict glycemic controls, symptom control through medication and therapy to improve quality of life and safety. Occupational and Physical therapy should be considered in all patients with moderate to severe $\mathrm{DN}$.

\section{Disease}

Diabetes is a growing concern of health professionals with rising numbers and an estimated $9.3 \%$ of the American population having the disease [1]. The direct and indirect cost of diabetes to US health care was $\$ 245$ billion dollars in 2012 and is only expected to increase [2]. Of these people, approximately $49 \%$ go on to develop some sort of neuropathy [3]. DN can greatly lower quality of life in diabetics and also increase mortality and morbidity [4-7]. The neuropathy can present in several ways; peripheral and symmetrical, focal, proximal and autonomic. Both type 1 and 2 diabetes have peripheral neuropathy as the most common but these neuropathies have also been noted in those with no diabetes but impaired glucose tolerance and prediabetics $[8,9]$.

\section{Pathology}

The pathogenesis behind diabetic neuropathy is multivariable. Hyperglycemia can cause neuropathy through production of reactive oxygen species, oxidative stress, inflammatory injury and advanced glycation end products [9]. Hyperlipidemia can also result in multiple mechanisms of neuronal injury. Free fatty acids can cause inflammatory cytokines, the fatty acids themselves have been shown to cause injury, and oxidized forms of these acids, LDL and other plasma proteins can trigger cascades that cause oxidative stress [10]. Insulin resistance has also been shown to downregulate signaling of neurotrophic growth factors [11]. Particular interleukin inflammatory proteins have been associated with large nerve sensorimotor neuropathy associated with IL-6 and IL-10 while IL-10 alone is implicated in demyelination [12]. The product of the aforementioned disruptions is a combination multiple cellular errors that can result in apoptosis, macrophage activation and loss of neurotrophic signals resulting in damage to the nerves manifesting in diabetic neuropathy [13].
The above intracellular stresses also happen to other cells, including muscle and nerves. Another large contributor to the neuropathy is the interruption of the neural microvasculature. Nerve biopsies show reduced oxygen tension, membrane thickening, endothelial cell proliferation and hypertrophy [14]. Pathology of diabetic amyotrophy affected nerves shows the same pattern: multifocal fiber loss, perineurial degeneration or thickening, neovascularization, and abortive regeneration of nerve fibers forming microfasciculi, and is significant for more ischemic damage than non-diabetic plexopathies [15]. In vivo studies on neuronal blood flow have also shown abnormalities and slower flow on diabetic patients with peripheral neuropathy compared to diabetics with no neuropathy [16].

This microvascular damage causes the manifestation of a significant amount of the other symptoms including but not limited to nephropathy and retinopathy, as well as myopathic changes [17]. The muscle is also directly weakened by the intracellular damage but also the loss of innervation and both macro and microvasculature damage, leading to atrophy, weakness, fatigue and slowing of contraction [18]. Patients with DN have been shown to have accelerated loss of strength, mass and quality of muscles compared to diabetics without neuropathy [19]. This simultaneous damage of sensation and strength greatly increases morbidity [20].

\section{Presentation}

Peripheral neuropathy is the classic, most common diabetic neuropathy. It is length dependent, striking the feet first in a stocking type pattern. There is a gradual and insidious multimodal loss of sensation that can present with a variety of combination of complaints including electric stinging, hyperanalgesia, burning, numbness, and imbalance in addition to other diabetic complications [21]. Physical signs of extensor digitorum brevis wasting, loss of ankle reflexes, foot drop and hair loss can be observed [13]. This can progress to a point to having motor nerves and upper extremities also involved in severe

*Corresponding author: Aiesha Ahmed, MD, Department of Neurology, Penn State University, 30 Hope Drive, EC 037 Hershey, PA 17033, USA, Tel: 7175311377 ; Fax: 7175310384; E-mail: aahmed1@hmc.psu.edu

Received November 30, 2016; Accepted December 07, 2016; Published December 14, 2016

Citation: Jens W, Ahmed A (2016) Peripheral Nervous System Involvement in Diabetes and Role of Rehabilitation. Int J Neurorehabilitation 3: 233. doi: 10.4172/2376-0281.1000233

Copyright: @ 2016 Jens W, et al. This is an open-access article distributed under the terms of the Creative Commons Attribution License, which permits unrestricted use, distribution, and reproduction in any medium, provided the original author and source are credited. 
cases. The loss of pedal sensation and places the patient at risk for foot ulcers and falls.

True entrapment presents with slow progression and long lasting, requiring intervention differs from mononeurtis which presents acutely with pain and is self-limited in the distribution of the affected nerve [22].

Mononeuritis is called by vasculitis and ischemia to the nerve and is associated with weight loss. Mononeuritis and entrapment both affect similar nerves, including cranial (III, IV, VI, VII), in addition to peripheral peroneal, sacral, femoral, median and ulnar, with median as the most common [23]. True entrapment is also worsened in diabetes, up to $3 \mathrm{x}$ the normal population, which is thought to be increased damage to areas of compression, poorer recovery to damage, metabolic changes and swelling [24]. Each focal neuropathy will present with loss of function and perhaps pain or tingling in same area. Multifocal neuropathy (aka mononeuritis multiplex) can also occur, with at least 2 nerves independently affected, presenting with asymmetric neuropathy with pain and similar to mononeuritis, be associated with weight loss [25].

While DN can show diffuse neuronal injury, the one of the rarer but concerning sites are the major nerve plexuses when it manifests as proximal diabetic neuropathy (aka Diabetic amyotrophy, diabetic cervical radiculoplexus neuropathy, diabetic thoracic radiculoneuropathy, and diabetic lumbosacral radiculoplexus neuropathy). The brachial plexus originates from C5-T1 in $95 \%$ of individuals with occasional anomalous fibers originating from $\mathrm{C} 4$ and $\mathrm{T} 2$ and can be separated into supraclavicular, retroclavicular, and infraclavicular regions. The supraclavicular region is made up of the roots and trunks, with $\mathrm{C} 5$ and $\mathrm{C} 6$ joining to give the upper trunk; C7 becomes the middle trunk and C8-T1 joining to become the lower truck. Below the clavicle, there trunks split into anterior and posterior divisions and recombine below the clavicle to become the 3 cords named in according to their position around the subclavian artery. These then become the 5 terminal branches. Along the entire structure, eleven minor nerves exit at particular locations, which greatly aid in localization. The lumbosacral plexus originates from the anterior divisions of the lumbar, sacral, and coccygeal nerve, with occasional supplementation from T12. It is well protected in the retroperitoneal space which makes it less prone to injury than the exposed brachial plexus. The major divisions are the obturator and femoral nerve from L2-4 lumbar plexus and the sciatic nerve from L4-S2 from the lumbosacral plexus which further separates to the tibial and peroneal nerves.

Diabetic Autonomic neuropathy (DAN) is one of the greater concerns of $\mathrm{DN}$; given it is a significant source of morbidity and mortality [7]. Major clinical symptoms of DAN include neurovascualar dysfunction, sexual dysfunction, neurogenic bladder, sudomotor dysfunction, orthostatic hypotension, gastroparesis, exercise intolerance and even worsened control of glucose ("brittle diabetes"). $25 \%$ of type 1 and $32 \%$ of type 2 diabetics have been shown to have two or more autonomic abnormalities on testing [26]. Cardiac autonomic neuropathy has a 5 times increase in mortality, compared to those without [27]. Sadly it is also the most common nerve affected in DAN, given the vagal nerve is the longest parasympathetic nerve and the length dependent nature of the disease [27]. Unfortunately, DAN is often overlooked and not tested for despite the impact on quality of life [28].

\section{Work Up}

Establishing the neuropathy is solely related to diabetes and not a severe or correctable cause is an important step, as $10 \%$ of neuropathies in diabetics have been shown to have alternate causes [29]. Given the likely underlying pathology, glucose testing including hemoglobin A1c (HbAlc), fasting blood glucose, and possible glucose tolerance test in those that test normal for HbAlc show be obtained. AAN guidelines also recommend B12 with methylmalonic acid and or homocysteine as well as protein electrophoresis [30]. Further testing is reliant on a clinician's judgement; is family history concerning for a genetic disorder, are there other autoimmune symptoms, or previous history of/or concern for cancer are among questions that should be addressed and considered. In proximal neuropathy, laboratory studies in the disease can be variable with nonspecific inflammatory findings. A small study showed that CSF protein was elevated in $90 \%$ of subjects with ESR/CRP raised in 33\% and other ANA/ANCA and RF findings elevated in $25 \%$ [31].

Both Electromyography (EMG) and nerve biopsy have good negative predictive value at ruling out diabetic peripheral neuropathy but poor positive predictive value, and their utility is best used to give supportive information to the clinical diagnosis or rule out an alternate pathology [30,32]. Dyck et al. suggests the use of two abnormalities in the following fibular motor nerve conduction velocity (MNCV), sural sensory nerve action potential, tibial MNCV, ulnar MNCV, tibial F-wave latency and ulnar F-wave latency as an acceptable in supporting diabetic peripheral neuropathy [33]. Given the relatively low diagnostic yield in a clear case of peripheral DN, EMG should be reserve for more atypical presentations and in cases concerning focal or proximal neuropathy. EMG in focal neuropathy can establish and confirm the nerve in question is impaired and others are relatively spared. In multifocal diabetic neuropathy, EMG will show a focal conduction block over short nerve segments away from entrapment sites, as illustrated by the loss of compound motor action potential amplitude across the block but preserved amplitude before and after the lesion [34].

Nerve conductive studies of proximal neuropathy are typically abnormal, however four weeks should be allowed for the emergence of the symptoms to permit EMG changes develop. Electomyographic changes are also related to timing with initial proximal muscles involvement with distal evidence appearing later, with a length dependent axonal general sensorimotor polyneuropathy [35]. Sensory responses can be absent or reduced with evidence of axonal loss as illustrated by reduced compound action potential amplitudes and proportionate slowing of conduction velocities [36]. Acute needling can reveal positive sharp waves, fibrillations and reduced recruitment of voluntary motor action potentials, while chronic cases will reflect neuropathic changes of increased amplitude and duration with reduced recruitment and polyphasic waves [35]. Care should also be taken in interpretation as up to $50 \%$ of patients with diabetic lumbosacral radiculoplexus neuropathy may have other simultaneous peripheral DN secondary to their underlying diabetes [37].

DAN can be confirmed by using the composite autonomic scoring scale; composed of QSART sweat test, orthostatic vitals, heart rate response to tilt and deep breathing, the valsava ratio, and beat to beat $\mathrm{BP}$ measurements during a variety of movements [38]. This composite is a specific and sensitive way to assess autonomic dysfunction [38]. Other test can be used to assess the presence of autonomic dysfunction in other organ systems, including penile tumescence studies, and vaginal plethysmography and other imaging studies discussed below [39].

While diabetes can have nonspecific findings of increased cerebral microvascular disease and smaller cross sections of spinal cords, imaging is typically not indicated in peripheral DN or focal neuropathy unless another etiology is in the suspected after a thorough history and 
physical exam [40]. Imaging of the brachial and lumbrosacral plexuses is indicated in assessing proximal neuropathy, as it is a diagnosis of exclusion. MRI with $1.5 \mathrm{~T}$ or greater magnet is the preferred modality for non-traumatic brachial pathology and will help rule out other etiologies possible in the diagnosis of proximal neuropathy, including compression, malignancy, musculoskeletal disease, infection and inflammatory disorders [41]. The diffusion weighted image with high diffusion sensitizing gradient movements helps highlight the nerve bundles allowing for the best visualizations of pathology [42]. A nonspecific pattern of increased T2, Gadolinium enhancement and/ or nerve enlargement can be observed in proximal neuropathy [43]. Imaging in DAN includes ways to assess the functioning of the organ systems, including gastric emptying studies, post void bladder scans, as well as 123-iodine metaiodobenzylguanidine scans which evaluate the sympathetic innervation of the heart, and loss is a strong predictor of heart failure [44].

\section{Medical Management}

Glucose control is the primary goal in treatment. This prevents/ limits further damage to the neuromuscular system, however is much more effective in type 1 vs. type 2 [45]. Given the raised triglycerides and LDL levels in diabetes type 2, treatment with fenofibrates and statins can be considered if indicated [46]. Other options that limit end organ dysfunctions should also be considered, such as aspirin, blood pressure and lisinopril for kidney, heart, and vasculature protection. Multiple drugs and other compounds have been proposed in reducing or reversing $\mathrm{DN}$, however none have found to be successful in comprehensive reviews, with polyneuropathy with the anti-oxidant alpha-lipoic acid as a prime failed example [47]. Sadly, medical management in peripheral $\mathrm{DN}$ is reduced to controlling the underlying illness and managing symptoms.

Symptom management of neuropathic pain includes use of pregabalin as class A evidence and selective norepinephrine reuptake inhibitors, Tricyclic antidepressants, gabapentin, with opiates considered in the acute phase as class b evidence [48]. This slightly varies from a 2010 Cochrane report indicating Tricyclics being the initial medication based on lowest number needed to treat (1.3-2.4), with pregabalin next, followed by SNRI and gabapentin [49]. Selective serotonin reuptake inhibitors can help treat with the pain and depression aspects of the disease [40]. Topical medications from over the counter capsaicin to prescription lidocaine or elaborate compounded mixtures are also indicated [48].

Focal neuropathy treatment varies depending on etiology. Entrapment neuropathy can initially undergo anti-inflammatory trials and bracing, however if it persists or the EMG is supportive of moderate or severe damage, surgical release may be necessary. Mononeuritis is self-limited and pain control is the only goal, as recovery should occur within the year. There is anecdotal evidence in the literature for mononeuritis to be treated with immunosuppression but it remains an area where additional research is needed [50].

While the approach for treatment for proximal DN is now more accepted, initially there was a vibrant debate. There was a vocal community who believed that given the inflammatory markers, the perivascular inflammation on pathology and a presentation similar to chronic inflammatory demyelinating polyneuropathy; immunosuppression was the treatment of choice [51]. Studies and case series appeared to show benefit of chronic immunosuppression and even intravenous immunoglobulins where used [51-53]. However, in 2009 and again in 2012, the Cochrane Review found no evidence in review of all the studies for the role of immunosuppression in DA, with the caveat that high dose steroids was attempted in only a small subgroup and unable to comment with that modality [54]. Unfortunately, no multicenter trial has been completed and no cohesive recommendations beside symptom management and strict glucose control.

DAN treatment is again more symptom management. For cardiac DAN, beta blockers to control tachycardia, lisinopril for blood pressure and remodeling and TEDs, abdominal binders and midodrine/ fludrocortisone for hypotension are some of the various options, in addition to life style changes and therapy [55]. Medications or devices to assist with erections in men or lubrication for women as well as sex therapy are options for Diabetes induced sexual dysfunction. Gastric autonomic treatment is broad but roughly equates to stimulating movement (prokinetic agents, limiting meal size, bulking agents, botox to sphincters), symptom control (anti-nausea, Proton pump inhibitors) or bypassing the system with tube feedings [55]. Other systems treatments may include bethamachol or intermittent catheterization for diabetic bladder and driving restrictions for pupil amotility [56].

\section{Rehabilitation}

Given the medical management of DN is limited to salvaging the remaining nerves and treating symptoms, a more rounded approach should be considered. Comprehensive medical management and multiple modalities, especially a trained nurse educator and coordinator are effective in improving the process of care in diabetes [57]. Physical therapy (PT), Occupational Therapy (OT), and Nutritional Therapy (NT) should be considered in each patient to maximize treatment and help advise on the "diet and exercise" potion of DN treatment.

NT is an important consideration for diabetics, given diet's obvious role in the disease. Focused intervention on nutritional education and providing recipes and ways to improve a patients diet have been shown to have excellent results in reducing BMI, waist circumference and $\mathrm{HbAlc}$ [58]. An individualized nutritional approach is also recommended as it appears to promote better adherence [59]. In cases of impaired gastric motility, the nutritionist can help advise on alterations in diet to improve fiber and flow and, in extreme cases requiring tube feeding, knowledge and recommendations on enteral feeding.

Physical therapy can help develop an appropriate exercise program for patients with limitations. Exercise of more than 150 min per week has been shown to help improve $\mathrm{HbA1c}$ with diet changes [60]. Exercise itself has been shown to help increase neurotrophic and vasculotrophic signals and can improve DN symptoms and promote the re-growth of small nerve fibers [61]. Joint function and strength can be preserved and/or recovered in DN with range of motion and strengthening exercises. Therapist can also assist tin the assessment in the need of therapeutic devices such as wheelchairs, cane and cane-variety, braces and splints to help preserve the patient's mobility.

Given the combination of diabetes on gait and safety, gait training in diabetes in a potential way to reduce falls in DN. Gait could be affected in three possible levels, peripheral secondary myopathy, DPN and DAN, spinal cord to brainstem resulting from small vessel strokes causing loss of sensory information and coordination, and cortical/subcortical lesions from DM related strokes, neurodegenerative diseases and small vessel disease causing higher level gait apraxia [62]. Specific advice on lifestyle choices as well as training has been shown to improve reaction time, postural balance and gait speed [63]. Directed physical therapy to the ankle to help reduce rollovers and improve stability [64] 
Pain treatment is an overlooked aspect which physical therapy can assist the physician. Transcutaneous electrical nerve stimulation stimulate nerves topically with electric current. The signal generates contractions and heat that serves to relieve stiffness, improve mobility and relieve pain and has been shown to be an effective adjunctive therapy in diabetes management [65]. Interferential therapy uses the strong physiological effects of low frequency electrical stimulation of nerves. Ultrasound and other modalities have also been used to assist in pain control in $\mathrm{DN}$.

Occupational therapy's goal is to maintain functional independence by educating and training patients how to best use adaptive techniques and strategies of activities of daily living. These trainings can be done to improve skills at home, work or generally. Home or work evaluations for safety and need for assistive devices and technology should be performed. The occupational therapists are also good at establishing goals and assisting with carrying out a comprehensive plan, helping compliance and helping coordinate care.

\section{Conclusion}

We have above given an overview of the diabetic peripheral nervous system clinical presentations which are diverse. Pathophysiology varies as well based on the presentation. The goal of the management is multidisciplinary and the important role of different rehabilitation modalities is discussed to provide guideline to clinicians from different specialties who work with such patients.

\section{References}

1. Centers for Disease Control and Prevention (2014) National diabetes statistics report: Estimates of diabetes and its burden in the United States, 2014. US Department of Health and Human Services, Atlanta, GA.

2. American Diabetes Association (2013) Economic costs of diabetes in the U.S in 2012. Diabetes Care 36: 1033-1046.

3. Abbott CA, Malik RA, van Ross ER, Kulkarni J, Boulton AJ (2011) Prevalence and characteristics of painful diabetic neuropathy in a large community-based diabetic population in the U.K. Diabetes Care 34: 2220-2224.

4. Argoff CE, Cole BE, Fishbain DA, Irving GA (2006) Diabetic peripheral neuropathic pain: Clinical and quality-of-life issues. Mayo Clin Proc 81: S3-S11.

5. Margolis DJ, Malay DS, Hoffstad OJ, Leonard CE, MaCurdy T, et al. (2011) Incidence of diabetic foot ulcer and lower extremity amputation among Medicare beneficiaries, 2006 to 2008: Data Points \#2.

6. Agrawal Y, Carey JP, Della Santina CC, Schubert MC, Minor LB (2010) Diabetes, vestibular dysfunction and falls: Analyses from the National Health and Nutrition Examination Survey. Otol Neurotol 31: 1445-1450.

7. Soedamah-Muthu SS, Chaturvedi N, Witte DR, Stevens LK, Porta M, et al. (2008) EURODIAB Prospective Complications Study Group. Relationship between risk factors and mortality in type 1 diabetic patients in Europe: the EURODIAB Prospective Complications Study (PCS). Diabetes Care 31: 1360-1366.

8. Tracy JA, Dyck PJ (2008) The spectrum of diabetic neuropathies. Physical medicine and rehabilitation clinics of North America 19: 1-v.

9. Vincent AM, Callaghan BC, Smith AL, Feldman EL (2011) Diabetic neuropathy: Cellular mechanisms as therapeutic targets. Nat Rev Neurol 7: 573-583.

10. Wiggin TD, Sullivan KA, Pop-Busui R, Amato A, Sima AA, et al. (2009) Elevated triglycerides correlate with progression of diabetic neuropathy. Diabetes 58 : 1634-1640.

11. Kim B, Feldman EL (2012) Insulin resistance in the nervous system. Trends Endocrinol Metab 23: 133-141.

12. Magrinelli F, Briani C, Romano M, Ruggero S, Toffanin E, et al. (2015) The association between serum cytokines and damage to large and small nerve fibers in diabetic peripheral neuropathy. J Diabetes Res 23: 133-141.

13. Callaghan BC, Cheng HT, Stables CL, Smith AL, Feldman EL (2012) Diabetic neuropathy: Clinical manifestations and current treatments. Lancet Neurol 11 521-534
14. Malik RA, Tesfaye S, Thompson SD, Veves A, Sharma AK, et al. (1993) Endoneurial localisation of microvascular damage in human diabetic neuropathy. Diabetologia 36: 454-459.

15. Dyck PJ, Engelstad J, Norell J, Peter J (2000) Dyck: Microvasculitis in nondiabetic lumbosacral radiculoplexus neuropathy: Similarity to the diabetic variety (DLSRPN). J Neuropathol Exp Neurol 59: 525-538.

16. Tesfaye S, Harris N, Jakubowski JJ, Mody C, Wilson RM, et al (1993) Impaired blood flow and arterio-venous shunting in human diabetic neuropathy: A nove technique of nerve photography and fluoroscein angiography. Diabetologia 36 1266-1274.

17. Forbes JM, Cooper ME (2013) Mechanisms of diabetic complications. Physiol Rev 93: 137-188.

18. Allen MD, Doherty TJ, Rice CL, Kimpinski K (2016) Physiology in medicine: Neuromuscular consequences of diabetic neuropathy. J Appl Physiol 121: 1-6.

19. Andersen $H$ (2012) Motor dysfunction in diabetes. Diabetes Metab Res Rev 28 Suppl 1: 89-92.

20. Orlando G, Balducci S, Bazzucchi I, Pugliese G, Sacchetti M (2016) Neuromuscular dysfunction in type 2 diabetes: Underlying mechanisms and effect of resistance training. Diabetes Metab Res Rev. 32: 40-50.

21. Boulton AJ (2005) Management of diabetic peripheral neuropathy. Clinical Diabetes 23: 9-15.

22. Wilbourn AJ (1999) Diabetic entrapment and compression neuropathies Diabetic Neuropathy 481-501.

23. VinikA, Mehrabyan A, Colen L, Boulton A (2004) Focal entrapment neuropathies in diabetes. Diabetes Care 27: 1783-1788.

24. Chaudhuri KR, Davidson AR, Morris IM (1989) Limited joint mobility and carpa tunnel syndrome in insulin-dependent diabetes. Br J Rheumatol 28: 191-194.

25. Kelkar P, Parry GJ (2003) Mononeuritis multiplex in diabetes mellitus: Evidence for underlying immune pathogenesis. J Neurol Neurosurg Psychiatry 74: 803806.

26. Ziegler D, Gries FA, Spuler M, Lessmann F (1992) Diabetic cardiovascular autonomic neuropathy multicenter study group: The epidemiology of diabetic neuropathy. J Diabetes Complications 6: 49-57.

27. Ziegler D (1999) Cardiovascular autonomic neuropathy: Clinical manifestations and measurement. Diabetes Reviews 7: 300-315.

28. Chyun DA, Wackers FJt, Inzucchi SE, Jose P, Weiss C, et al. (2015) Autonomic dysfunction independently predicts poor cardiovascular outcomes in asymptomatic individuals with type 2 diabetes in the DIAD study. SAGE Open Medicine 3: 2050312114568476.

29. Dyck PJ, Katz KM, Karnes JL, Litchy WJ, Klein R, et al. (1993) The prevalence by staged severity of various types of diabetic neuropathy, retinopathy and nephropathy in a population-based cohort: the Rochester Diabetic Neuropathy study. Neurology 43: 817-824.

30. England JD, Gronseth GS, Franklin G, Carter GT, Kinsella LJ, et al. (2009) Practice parameter: The evaluation of distal symmetric polyneuropathy: The role of autonomic testing, nerve biopsy and skin biopsy (an evidence-based review) Report of the American Academy of Neurology, the American Association of Neuromuscular and Electrodiagnostic Medicine and the American Academy of Physical Medicine and Rehabilitation. PMR 1: 14-22.

31. Massie R, Mauermann ML, Nathan PS, Kimberly KA, Dyck PJ, et al (2012) Diabetic cervical radiculoplexus neuropathy: A distinct syndrome expanding the spectrum of diabetic radiculoplexus neuropathies. Brain 135: 3074-3088.

32. Smith A, Lessard M, Singleton J (2015) The diagnostic utility of nerve conduction studies and skin biopsy for diabetic neuropathy (DPN): A Bayesian analysis. Neurology 84: 7 .

33. Dyck PJ, Carter RE, Litchy WJ (2011) Modeling nerve conduction criteria for diagnosis of diabetic polyneuropathy. Muscle Nerve 44: 340-345.

34. Bromberg MB (2013) An electrodiagnostic approach to the evaluation of peripheral neuropathies. Physical Medicine and Rehabilitation Clinics of North America 24: 153-168.

35. Bastron JA, Thomas JE (1981) Diabetic polyradiculopathy: Clinical and electromyographic findings in 105 patients. Mayo Clin Proc 56: 725-732. 
Citation: Jens W, Ahmed A (2016) Peripheral Nervous System Involvement in Diabetes and Role of Rehabilitation. Int J Neurorehabilitation 3: 233. doi: $10.4172 / 2376-0281.1000233$

36. Dyck PJ, Windebank AJ (2002) Diabetic and non-diabetic lumbosacral radiculoplexus neuropathies: New insights into pathophysiology and treatment. Muscle Nerve 25: 477-491.

37. Bhanushali MJ, Muley SA (2008) Diabetic and non-diabetic lumbosacral radiculoplexus neuropathy. Neurol India 56: 420-425.

38. Low PA (1993) Composite autonomic scoring scale for laboratory quantification of generalized autonomic failure. Mayo Clin Proc 68: 748-752.

39. Vinik Al, Maser RE, Mitchell BD, Freeman R (2003) Diabetic autonomic neuropathy. Diabetes Care 26: 1553-1579.

40. Tesfaye S, Selvarajah D (2012) Advances in the epidemiology, pathogenesis and management of diabetic peripheral neuropathy. Diabetes Metab Res Rev 28 Suppl 1: 8-14.

41. Amrami KK, Port JD (2005) Imaging the brachial plexus. Hand Clin 21: 25-37.

42. Martinoli C, Gandolfo N, Perez MM, Klauser A, Palmieri F, et al. (2010) Brachial plexus and nerves about the shoulder. Semin Musculoskelet Radiol. 14: 523-546.

43. Delaney H, Bencardino J, Rosenberg ZS (2014) Magnetic resonance neurography of the pelvis and lumbosacral plexus. Neuroimaging Clin $\mathrm{N} \mathrm{Am}$ 24: $127-150$

44. Boogers JM, Veltman EC, Bax JJ (2011) Cardiac autonomic nervous system in heart failure: Imaging technique and clinical implications. Curr Cardiol Rev 7: $35-42$

45. Ismail-Beigi F, Craven T, Banerji MA, Basile J, Calles J, et al. (2010) Effect of intensive treatment of hyperglycaemia on microvascular outcomes in type 2 diabetes: An analysis of the ACCORD randomised trial. Lancet 376: 419-430.

46. Smith AG, Singleton JR (2013) Obesity and hyperlipidemia are risk factors for early diabetic neuropathy. J Diabetes Complications 27: 436-442.

47. Ziegler D, Hanefeld M, Ruhnau KJ, Hasche H, Lobisch M, et al. (1999) Treatment of symptomatic diabetic polyneuropathy with the antioxidant alphalipoic acid: A 7 month multicenter randomized controlled trial (ALADIN III Study). ALADIN III Study Group. Alpha-Lipoic Acid in Diabetic Neuropathy. Diabetes Care 22: 1296-1301

48. Bril V, England J, Franklin GM, Backonja M, Cohen J, et al. (2011). Evidencebased guideline: Treatment of painful diabetic neuropathy: Report of the American Academy of Neurology, the American Association of Neuromuscular and Electrodiagnostic Medicine and the American Academy of Physical Medicine and Rehabilitation. Neurology 3: 345-352.

49. Saarto T, Wiffen PJ (2010) Antidepressants for neuropathic pain: A cochrane review. J Neurol Neurosurg Psychiatry 81: 1372-1373.

50. Abgral S, Mouthon L, Cohen P, Guillevin L (2001) Localized neurological necrotizing vasculitides: Three cases with isolated mononeuritis multiplex. J Rheumatol 28: 631-633.
51. Verma A, Bradley WG (1994) High-dose intravenous immunoglobulin therapy in chronic progressive lumbosacral plexopathy. Neurology 44: 248-250.

52. Krendel DA, Costigan DA, Hopkins LC (1995) Successful treatment of neuropathies in patients with diabetes mellitus. Arch Neurol 52: 1053-1061.

53. Younger DS, Rosoklija G, Hays AP, Trojaborg W, Latov N (1996) Diabetic peripheral neuropathy: A clinicopathologic and immunohistochemical analysis of sural nerve biopsies. Muscle Nerve 19: 722-727.

54. Chan YC, Lo YL, Chan ES (2009) Immunotherapy for diabetic amyotrophy Cochrane Database Syst Rev 6: CD006521.

55. Deli G, Bosnyak E, Pusch G, Komoly S, Feher G (2013) Diabetic neuropathies: Diagnosis and management. Neuroendocrinology 98: 267-280.

56. Kempler P, Amarenco G, Freeman R, Frontoni S, Horowitz M, et al. (2011) Management strategies for gastrointestinal, erectile, bladder and sudomotor dysfunction in patients with diabetes. Diabetes Metab Res Rev 27: 665-677.

57. Renders CM, Valk GD, Griffin SJ, Wagner EH, Eijk Van JT, et al. (2001) Interventions to improve the management of diabetes in primary care, outpatient and community settings: A systematic review. Diabetes Care 24: 1821-1833.

58. Coppell KJ, Kataoka M, Williams SM, Chisholm AW, Vorgers SM, et al. (2010) Nutritional intervention in patients with type 2 diabetes who are hyperglycaemic despite optimised drug treatment-Lifestyle over and above drugs in diabetes (LOADD) study: Randomised controlled trial. BMJ 341: c3337.

59. Franz MJ, Boucher JL, Evert AB (2014) Evidence-based diabetes nutrition therapy recommendations are effective: The key is individualization. Diabetes Metab Syndr Obes 7: 65-72.

60. Umpierre D, Ribeiro PA, Kramer CK, Leitao CB, Zucatti AT, et al. (2011) Physical activity advice only or structured exercise training and association with $\mathrm{HbA} 1 \mathrm{c}$ levels in type 2 diabetes: A systematic review and meta-analysis. JAMA 305: 1790-1799.

61. Singleton JR, Smith AG, Marcus RL (2015) Exercise as therapy for diabetic and prediabetic neuropathy. Curr Diab Rep 15: 120.

62. Mayne D, Stout NR, Aspray TJ (2010) Diabetes, falls and fractures. Age Ageing 39: $522-525$

63. Allet L, Armand S, de Bie RA, Golay A, Monnin D, et al. (2010) The gait and balance of patients with diabetes can be improved: a randomised controlled trial. Diabetologia 53: 458-466.

64. Sartor CD, Hasue RH, Cacciari LP, Butugan MK, Watari R, et al (2014) Effects of strengthening, stretching and functional training on foot function in patients with diabetic neuropathy: results of a randomized controlled trial. BMC Musculoskelet Disord 15: 137.

65. Thakral G, Kim PJ, LaFontaine J, Menzies R, Najafi B, et al. (2013) Electrica stimulation as an adjunctive treatment of painful and sensory diabetic neuropathy. J Diabetes Sci Technol 7: 1202-1209. 